\title{
A multiple burst accretion Model to describe the metallicity distributions and stellar mass-metallicity relation for Local Dwarf Galaxies
}

\author{
F.D.A. Hartwick \\ Department of Physics and Astronomy, \\ University of Victoria, Victoria, BC, Canada, V8W $3 P 6$
}

\begin{abstract}
A one parameter model to describe the individual metallicity distributions and stellar mass-metallicity relation for dwarf galaxies is presented. This multipleburst model is based on an accretion scenario, accomodates the observational constraint between $\bar{z}$ and $\sigma_{z}^{2}$ recently established by Leaman (2012), and predicts a slope consistent with the stellar mass-metallicity relation of Kirby et al (2013) who showed that the local group dwarf spheroidal and dwarf irregular galaxies lie on the same relation. One interpretation of the model is that it describes star formation occuring either in gas rich mergers or at the intersection of colliding gas streams.
\end{abstract}

Subject headings: chemical evolution; dwarf galaxies

\section{Introduction}

Recently two important new results regarding the properties of dwarf galaxies have appeared. In one, Leaman (2012) showed that the same relation holds between the mean metallicity and the variance of the metallicity distributions for both the dwarf spheroidal and dwarf irregular galaxies. Secondly, Kirby et al. (2013) have shown that the local group dwarf spheroidal and dwarf irregular galaxies lie on the same stellar mass-metallicity relation. This suggests that a common evolutionary path exists for these two quite different morphological galaxy types. Clues to this evolutionary path must lie with the observed metallicity distributions. Unfortunately, the desired relationship is obscured because different evolutionary models can predict similar metallicity distributions e.g. Kirby et al. (2013) find that models with mass loss and those with accretion make quite similar predictions. Below we present an empirical model (the multiple- burst model) which accomodates the Leaman (2012) constraint, predicts the slope of the stellar mass-metallicity relation consistent with the value determined by Kirby et al.(2013) and is specified by only one parameter. 


\section{The Model}

Kirby et al (2013) and earlier work have shown that the observed $[\mathrm{Fe} / \mathrm{H}]$ distributions of the dwarf galaxies are nearly Gaussian-shaped functions with little or no metal poor tail. Both the simple closed box chemical evolution model (c.f. Searle and Sargent (1972), Pagel and Patchett (1975)) and the mass loss model of Hartwick (1976) predict a tail of metal poor stars which is more pronounced than in the above observations. Ways to limit the metal poor tail are to invoke either a pre-enrichment phase or an accretion model 1 (Kirby et al. 2013). Larson (1972) first introduced the idea of infall (or accretion) in order to limit the number of stars in the metal poor tail. Here, in addition to avoiding a metal poor tail, the desired model must maintain a body of enriched gas in order to account for the observations of the gas-rich dwarf irregular galaxies. We do this here by first generalizing the original Larson model. These results are then convolved with a metallicity kernel to produce the multiple-burst model. The metallicity kernel is specified in order to accomodate the Leaman (2012) metallicity constraint.

\subsection{Generalizing the Larson accretion model}

In the model of Larson (1972), star formation was assumed to occur at the same rate that gas was accreted. If $d M_{t}$ represents the incremental mass of gas accreted, and $d M_{s}$ and $d M_{g}$ represent the incremental mass of stars formed and gas accumulated respectively, then in the original model $d M_{s} / d M_{t}=1$ and $d M_{g} / d M_{t}=0$ i.e. $M_{g}=M_{g, 0}=$ constant.

Here we parameterize the star formation rate and gas accumulation rate as $d M_{s} / d M_{t}=$ $(1-q)$ and $d M_{g} / d M_{t}=q$ with $0<q<1$. When $q=0$, the Larson (1972) result is recovered but now with $q \neq 0$ the star formation rate can be lower than the accretion rate and enriched gas can be accumulated.

In the interest of keeping the equations simple in what follows we will assume that the symbols for metallicity $z$ and yield $p$ actually represent abundances with respect to the sun i.e. $z \equiv Z / Z_{\odot}$ and $p \equiv p / Z_{\odot}$.

The chemical evolution model used is the closed box model of Searle and Sargent (1972) and Pagel and Patchett (1975) where $M_{t}=M_{s}+M_{g}$ but here $d M_{t} \neq 0$ i.e.

$$
d z=1 / M_{g}\left[(p-z) d M_{t}-p d M_{g}\right]
$$

\footnotetext{
${ }^{1}$ The accretion model used by Kirby et al. was designed by Lynden-Bell (1975) to model the metallicity distribution of the local $\mathrm{G}$ - dwarfs.
} 
where $\mathrm{p}$ represents the true nuclear yield. The equation is solved for $M_{g}$ by substituting $d M_{g} / q$ for $d M_{t}$ and integrating. Solutions for $M_{t}$ and $M_{s}$ then follow.

$$
\begin{gathered}
M_{g}=M_{g, 0} /(1-z /((1-q) p))^{q} \\
M_{t}=M_{g, 0} /\left(q(1-z /((1-q) p))^{q}\right)-M_{g, 0}(1-q) / q
\end{gathered}
$$

and

$$
M_{s}=M_{t}-M_{g}=M_{g, 0}(1-q)\left(1 /(1-z /((1-q) p))^{q}-1\right) / q
$$

where we have assumed that the metallicity of the accreted gas is zero, and the initial amount of gas present is $M_{g, 0}$. The metallicity distribution is easily obtained by differentiating the above expressions. Whereas in the Larson (1972) model most of the stars formed have $z=p$, here most of the stars and the accumulated gas have $z=(1-q) p$. A more compact, parameterized solution to equation (1) is given by Binney \& Merrifield (1998).

The metallicity features predicted by the above model i.e. $d M_{s} / d z$ and $d M_{g} / d z$ look like $\delta$ functions and as such do not resemble the observed distributions. Rather these features resemble the result of an instantaneous star burst. In order to proceed we shall assume that star formation takes place in many smaller such bursts possibly as a result of small fluctuations in the accretion rate and whose amplitudes are specified by a metallicity kernel $f(z)$. The final distributions are then obtained by a convolution of this metallicity kernel with the above generalized Larson model results e.g. the star formation burst occurs at $z=(1-q) p$ and the relative mass of stars formed in this burst $d M_{s}$ is $(1-q) f(z) d z$. Similarly for the other two components i.e.

$$
\begin{aligned}
& d M_{s} / d z=(1-q) f(z)=z f(z) / p \\
& d M_{g} / d z=q f(z)=(1-z / p) f(z)
\end{aligned}
$$

and

$$
d M_{t} / d z=((1-q)+q) f(z)=f(z)
$$

The integration limits for all are $0 \rightarrow p$.

\subsection{The metallicity kernel}

The essence of this model is that chemical evolution evolution proceeds by repeated star bursts. The metallicity dependence is contained in the kernel. The perfect kernel needs to allow a description of the metallicity distributions while similtaneously providing a fit to the Leaman observations of $\bar{z}$ and $\sigma_{z}^{2}$ which is assumed to hold over the full range of $z$ and 
the stellar mass-metallicity relation. Both of the latter conditions are integral constraints. Leaman describes the overall metallicity distributions with only two parameters: the mean and the variance. This means that in general the kernel within the convolution integrals is not unique. The kernel chosen here attempts to accommodate all three of the above conditions while maintaining mathematical simplicity. It contains only one parameter and allows for relatively simple evaluation of the convolution integrals. Any fine tuning to improve the fits will likely require more than one parameter and additional mathematical complexity. The following expression for the kernel is adopted.

$$
f(z)=z^{2} e^{-z / a}
$$

Let the integrals of the moments of this function be represented as

$$
I_{n}=\int_{0}^{p} z^{n+1} e^{-z / a} d z=C_{n}\left(1-e^{-p / a}\left(1+g_{n}(p / a)\right)\right)
$$

where $C_{n}$ is a function of a only and $g_{n}(p / a)$ is a polynomial in $p / a$.

the mean and variance of the stellar component is given by

$$
\bar{z}=I_{3} / I_{2}
$$

and

$$
\sigma_{z}^{2}=I_{4} / I_{2}-\bar{z}^{2}
$$

Note that when $a \ll p$, the integrals can be expressed in terms of the constants $C_{n}$ only. Under this condition the results of the model simplify and the relation between $\bar{z}$ and $\sigma_{z}^{2}$ becomes $\log \sigma_{z}^{2}=2 \log \bar{z}-0.602$. The complete relation (for all $p / a$ ) is shown superimposed on the observational data of Leaman (2012) in Fig 1. Both here and in what follows we have assumed that the yield is $p=1$ i.e. the solar value. Generally this model requires that the true yield be at least as high or higher than the the metallicity of the metal richest star under consideration.

In order to account for the results in Fig. 1 Leaman (2012) has proposed a scenario based on the chemical evolution model of Oey (2000). While one can expect that recurrent star bursts will cause fluctuations in the accretion rate, the solution above is empirically motivated. No attempt is made here to model the separate relation found by Leaman (2012) for the star clusters.

The stochastic nature of the model (recurrent star bursts) suggests that a Gaussian kernel might be appropriate. Models with a Gaussian kernel were constructed following the procedures given here and the results are described in the appendix. 


\subsection{The model $[\mathrm{Fe} / \mathrm{H}]$ distributions}

It is common practise to compare model $[\mathrm{Fe} / \mathrm{H}]$ distributions with the observations in the $M_{s}-\log (z)$ plane and to identify distributions by $\mathrm{z}$ at the maximum $M_{s}$ and to refer to this quantity as the effective yield $p_{e f f}$. For the kernel we have chosen this quantity is $4 a$ i.e. $a=p_{\text {eff }} / 4$. From Eqn. (5) the appropriate distribution then becomes

$$
d M_{s} / d \log z=\ln (10) z^{4} e^{-z /\left(p_{e f f} / 4\right)} / p
$$

This distribution is shown as a dotted line superimposed on the histograms of the observations of three dwarf spheroidal galaxies and two dwarf irregulars in Figs 2-6. The data for the dwarf spheroidal galaxies comes from Kirby et al. (2010) and for the dwarf irregular galaxies from Kirby et al. (2013). The solid line in the figures is the dotted relation smoothed by a Gaussian kernel with the indicated $\sigma$ to show the effects of observational scatter. The fits are 'chi by eye'. All three distributions in each figure are similarly normalized. The fits are not perfect in that the model predicts a slight deficit of metal poor stars.

A related quantity of interest is the ratio of the mass in stars to the total mass accreted i.e. $M_{s} / M_{t}=I_{2} /\left(p I_{1}\right)$. When $a \ll p$ this ratio becomes $(3 / 4) p_{e f f} / p$.

\subsection{The accumulated gas $M_{g}$}

Enriched gas accumulates because not all the gas accreted gets turned into stars. Assuming that this gas is well mixed, the mean metallicity $\bar{z}_{M_{g}}$ is given by

$$
\bar{z}_{M_{g}}=\left(I_{2}-I_{3} / p\right) /\left(I_{1}-I_{2} / p\right)
$$

when $a \ll p$, this quantity approaches $\sim(3 / 4) p_{\text {eff }}\left(1-p_{\text {eff }} / 4 p\right)$ which is $\sim(3 / 4) \bar{z}_{M_{s}}$. It is reasonable to expect that some of this gas will be lost during the star bursts. However any that remains after the accretion phase ends should cool and as a result of any residual angular momentum form a disk. Stars may continue to form in this disk and if the object is sufficiently isolated will resemble a dwarf irregular galaxy. Dwarf spheroidal galaxies tend to be found as companions to a central galaxy and currently do not have any gas remaining. They must have lost their accumulated gas by some process such as ram-pressure stripping as they fall into and orbit the halo of the central galaxy. This does not preclude additional star formation having occured prior to or even during the gas removal process however. 


\subsection{The stellar mass-metallicity relation}

Kirby et al. (2013) have shown that the local dwarf spheroidal and dwarf irregular galaxies occupy the same stellar mass-metallicity relation. The relation between $\bar{z}$ and $M_{s}=I_{2} / p$ is easily constructed. When $a \ll p$ we note that $\log \bar{z}=\log a+\log 4$ and that $\log M_{s}=4 \log a-\log p+\log 6$ so that the slope of the relation is

$$
d \log \bar{z} / d \log M_{s}=0.25
$$

Kirby et al. (2013) find an observational slope of $0.30 \pm 0.02$. The value predicted by this simple model is in reasonable agreement with the observed value.

The model predicts a total baryon mass-metallicity relation with a logarithmic slope $\left(d \log \bar{z} / d \log M_{t}\right)$ of 0.33 when $a \ll p$.

\section{Discussion}

A multiple-burst accretion model of chemical evolution of dwarf galaxies is presented. The model can acount for the individual observed metallicity distributions, the observed mean z-variance relation, the observed stellar mass-metallicity relation and accounts for the distinction between dwarf spheroidal and dwarf irregular galaxies.

The purpose of constructing simple models such as the one above is twofold: it provides a useful analytical description of the observations and it may provide some insight into the much more complicated processes associated with galaxy formation and evolution. Given that the model is one of accretion suggests that star formation occurs incrementally with each incoming clump immediately turned into stars and enriched gas in a burst. This is in contrast with other models which start with a gas reservoir which is slowly converted into stars. The fact that not all of the gas is turned into stars suggests an inefficiency possibly the result of star formation occuring in gas cloud collisions rather than the less violent scenario of gravitational compression of gas within a stationary dark halo for example. The model may be describing what happens during early gas rich mergers or at the intersection of colliding gas streams. In fact this latter process could be responsible for the formation of the galaxies. More sophisticated modelling is required to validate the above interpretation. 


\section{Acknowledgements}

The author acknowledges financial support from an NSERC Discovery grant. He also thanks Adam Ritz for the use of his integral solver and Else Starkenburg and Ryan Leaman for discussions concerning the observations.

\section{Appendix}

As discussed above the stochastic nature of the model suggests that a Gaussian kernel may be appropriate as is the case if the central limit theorem was to apply. We outline the solution with it here.

Let the kernel be defined as

$$
f(z)=e^{-(z-a)^{2} / 2 b}
$$

The convolution integrals become

$$
I_{n}=\int_{0}^{p} z^{n-1} e^{-(z-a)^{2} / 2 b} d z
$$

To illustrate the general form of the integrals the expression for $I_{2}$ is given by

$$
I_{2}=a \sqrt{\pi b / 2}(\operatorname{erf}(a / \sqrt{2 b})-\operatorname{erf}((a-p) / \sqrt{2 b}))+b\left(e^{-a^{2} / 2 b}-e^{-(a-p)^{2} / 2 b}\right)
$$

where the error function $\operatorname{erf}(x)=\frac{2}{\sqrt{\pi}} \int_{0}^{x} e^{-t^{2}} d t$.

Letting $p_{\text {eff }}$ represent the maximum of the distribution in the $M_{s}-\log (z)$ plane requires the following relation between parameters a and $b$.

$$
p_{e f f}\left(p_{e f f}-a\right)-2 b=0
$$

Now let $a=\alpha p_{\text {eff }}$ so that $b=p_{\text {eff }}^{2}(1-\alpha) / 2$. Expressions for $\bar{z}$ and $\sigma_{z}^{2}$ can now be evaluated as above. The relation between $\log \sigma_{z}^{2}$ and $\log \bar{z}$ is well fit with $\alpha=0$ i.e. $a=0$ and $b=p_{\text {eff }}^{2} / 2$ and the result is almost identical to that shown in Fig 1 . When $p_{\text {eff }} / p \ll 1$ this relation is $\log \sigma_{z}^{2}=2 \log \bar{z}-0.563$.

With $\alpha=0$ and $p_{e f f} / p \ll 1$ the convolution integrals become very simple i.e $I_{1}=$ $p_{\text {eff }} \sqrt{\pi} / 2, I_{2}=p_{\text {eff }}^{2} / 2, I_{3}=p_{\text {eff }}^{3} \sqrt{\pi} / 4$, and $I_{4}=p_{\text {eff }}^{4} / 2$. Results in this regime follow easily i.e. $\bar{z}=p_{\text {eff }} \sqrt{\pi} / 2, \sigma_{z}^{2}=p_{\text {eff }}^{2}(1-\pi / 4), \bar{z}_{M_{g}} \sim p_{\text {eff }} / \sqrt{\pi}$ and $d \log \bar{z} / d \log M_{s}=0.50$. This last result for the logarithmic slope of the stellar mass-metallicity relation is significantly higher than the Kirby et al.(2013) result which is $0.30 \pm 0.02$. 
The $[\mathrm{Fe} / \mathrm{H}]$ distribution is easily calculated as

$$
d M_{s} / d \log z=\ln (10) z^{2} e^{-z^{2} /\left(p_{e f f}^{2}\right)} / p
$$

Fig. 7 shows this distribution calculated with the same parameters used in Fig.2. The two distributions are very similar although the Gaussian model is less symmetrical than the exponential model and may better account for the small metal poor tail.

In summary, the physically motivated Gaussian kernel model satisfies very well two of the three required conditions of the problem (the Leaman observations and the individual $[\mathrm{Fe} / \mathrm{H}]$ distributions) but not the logarithmic slope of the stellar mass-metallicity relation.

\section{REFERENCES}

Binney, J. \& Merrifield, M. 1998, Galactic Astronomy, (Princeton: Princeton University Press), 326

Hartwick, F. D. A. 1976, ApJ, 209, 418

Kirby, Evan N.; Guhathakurta, Puragra; Simon, Joshua D.; Geha, Marla C.; Rockosi, Constance M.; Sneden, Christopher; Cohen, Judith G.; Sohn, Sangmo Tony; Majewski, Steven R.; Siegel, Michael, 2010, ApJS, 191, 352

Kirby, Evan N.; Cohen, Judith G.; Guhathakurta, Puragra; Cheng, Lucy; Bullock, James S.; Gallazzi, Anna 2013, ApJ, 179, 102

Larson, R. B. 1972, Nature Phys. Sci., 236, 7

Leaman, R. 2012, AJ, 144, 183

Lynden-Bell, D. 1975, VA, 19,299

Oey, M. S. 2000, ApJ, 542, L25

Pagel, B. E. J. \& Patchett, B. E. 1975, MNRAS, 172, 13

Searle, L. \& Sargent, W. L. W. 1972, ApJ, 173, 25 


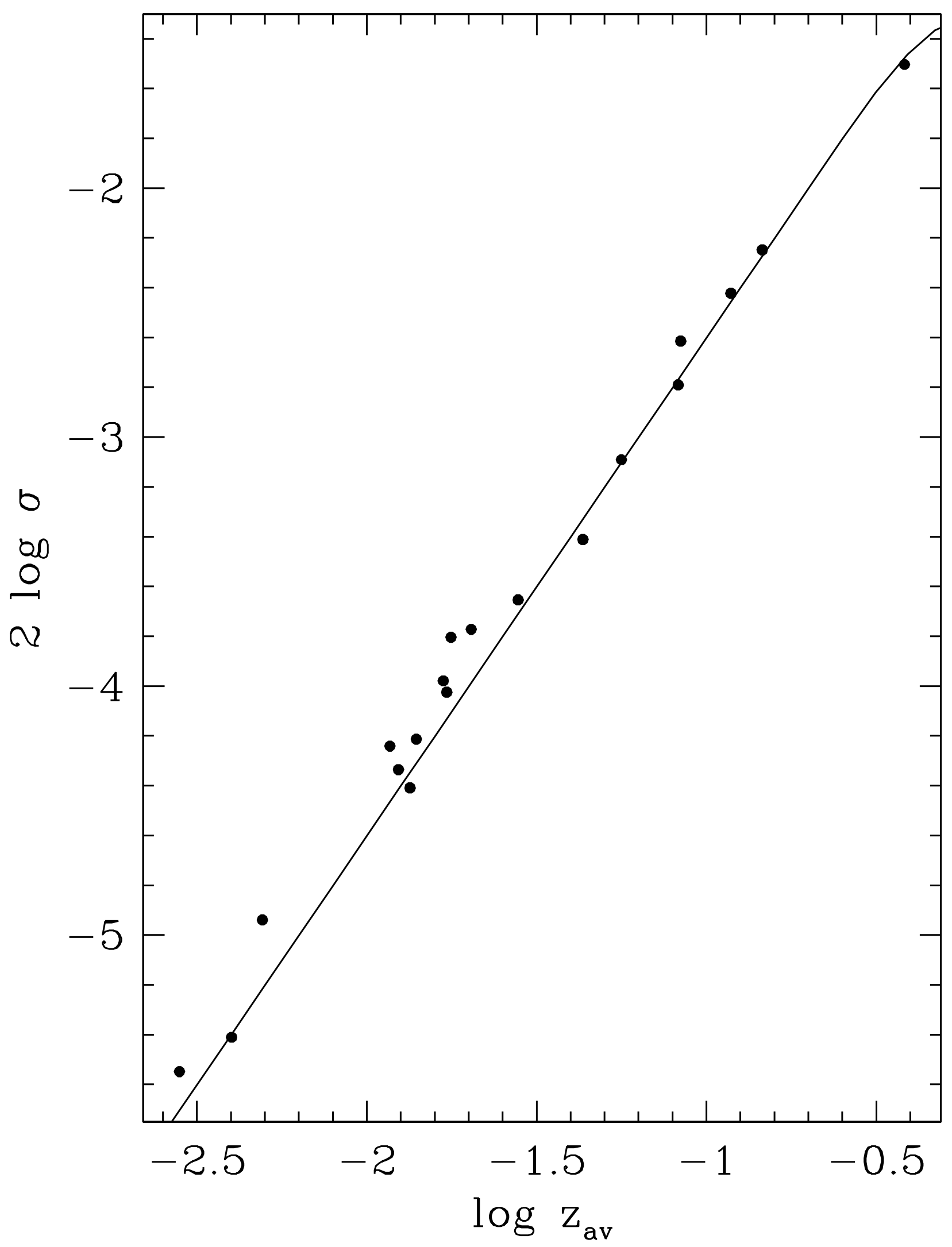

Fig. 1. - Points - the variance versus the mean metallicity of dwarf galaxies from Leaman (2012). The solid line is the relation calculated from the model. 


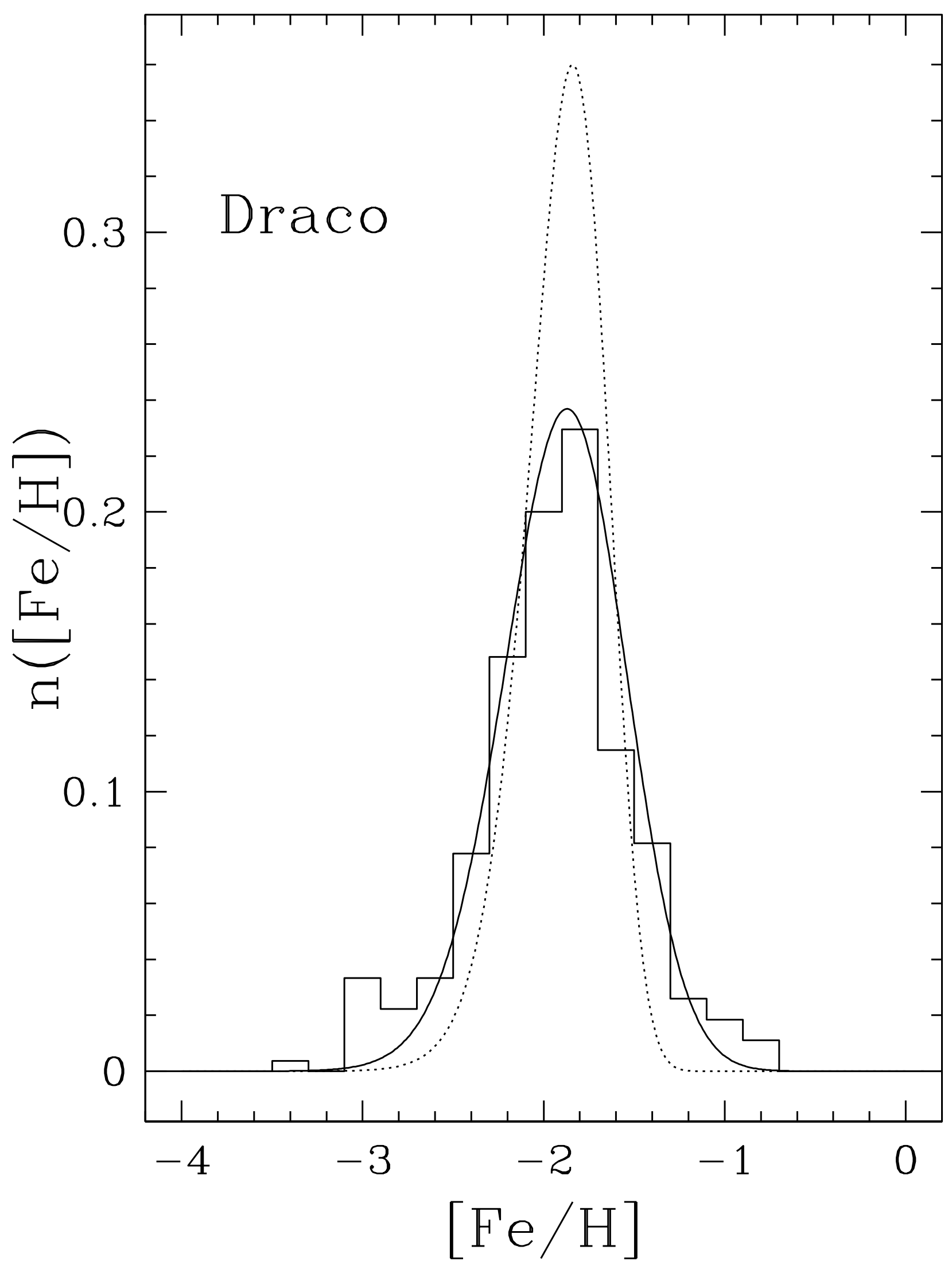

Fig. 2.- $[\mathrm{Fe} / \mathrm{H}]$ histogram for the Draco dwarf spheroidal galaxy. Dotted line - the model distribution with $\log p_{e f f}=-1.84$. Solid line - the model distribution smoothed by $\sigma=0.25$. 


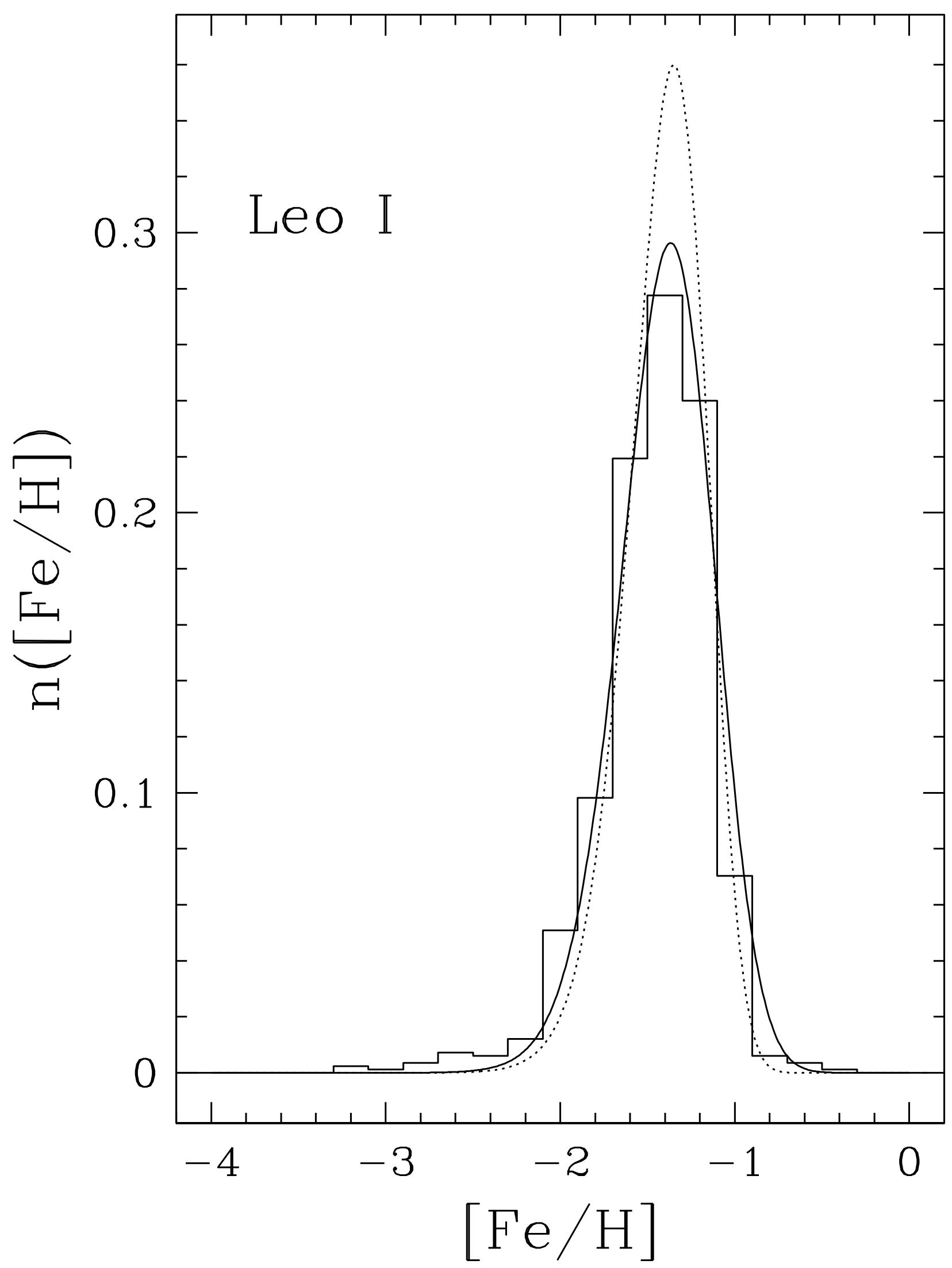

Fig. 3. $-[\mathrm{Fe} / \mathrm{H}]$ histogram for the Leo I dwarf spheroidal galaxy. Dotted line - the model distribution with $\log p_{e f f}=-1.35$. Solid line - the model distribution smoothed by $\sigma=0.15$. 


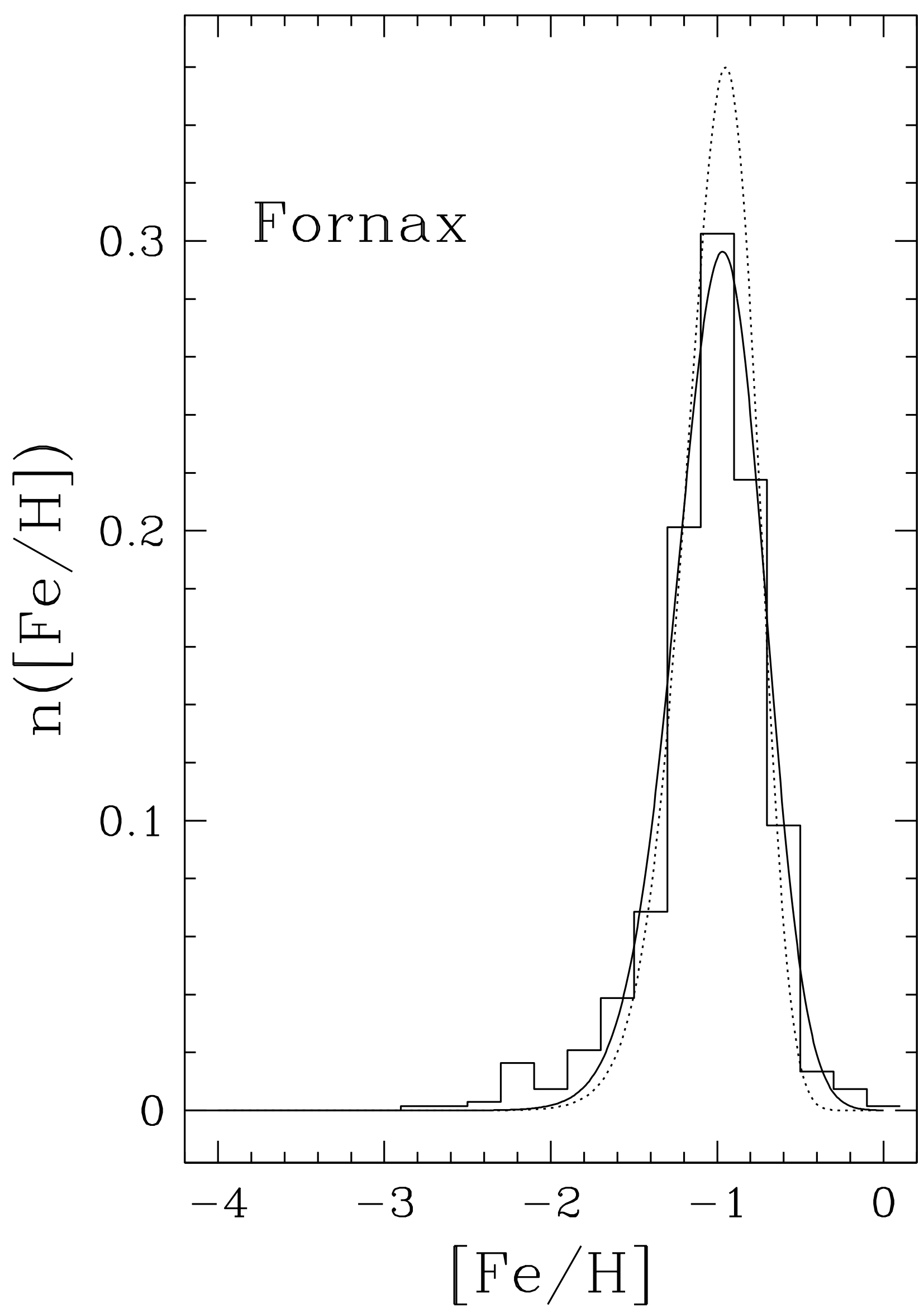




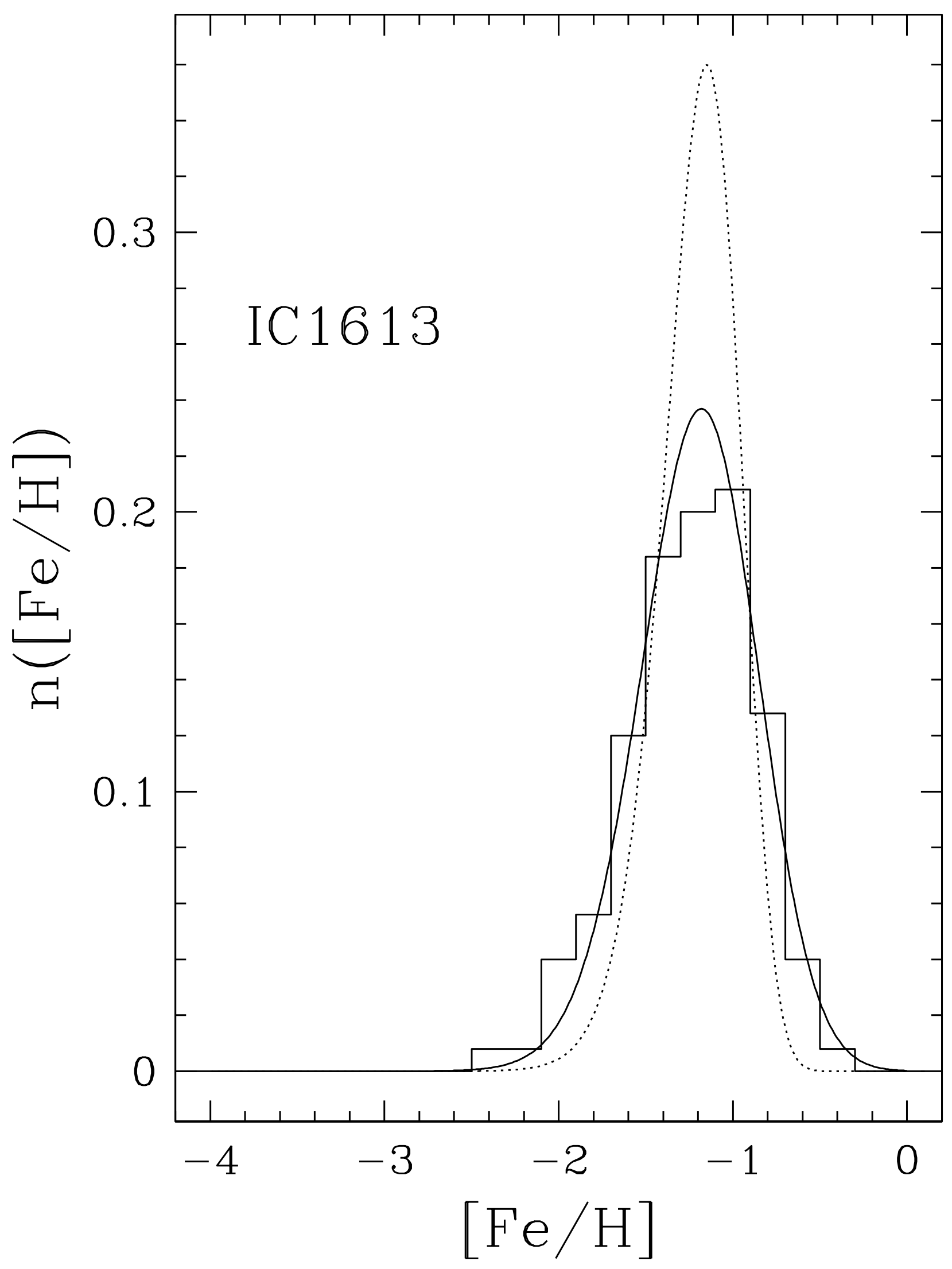

Fig. 5. $-[\mathrm{Fe} / \mathrm{H}]$ histogram for the dwarf irregular galaxy IC1613. Dotted line - the model distribution with $\log p_{\text {eff }}=-1.15$. Solid line - the model distribution smoothed by $\sigma=0.25$. 


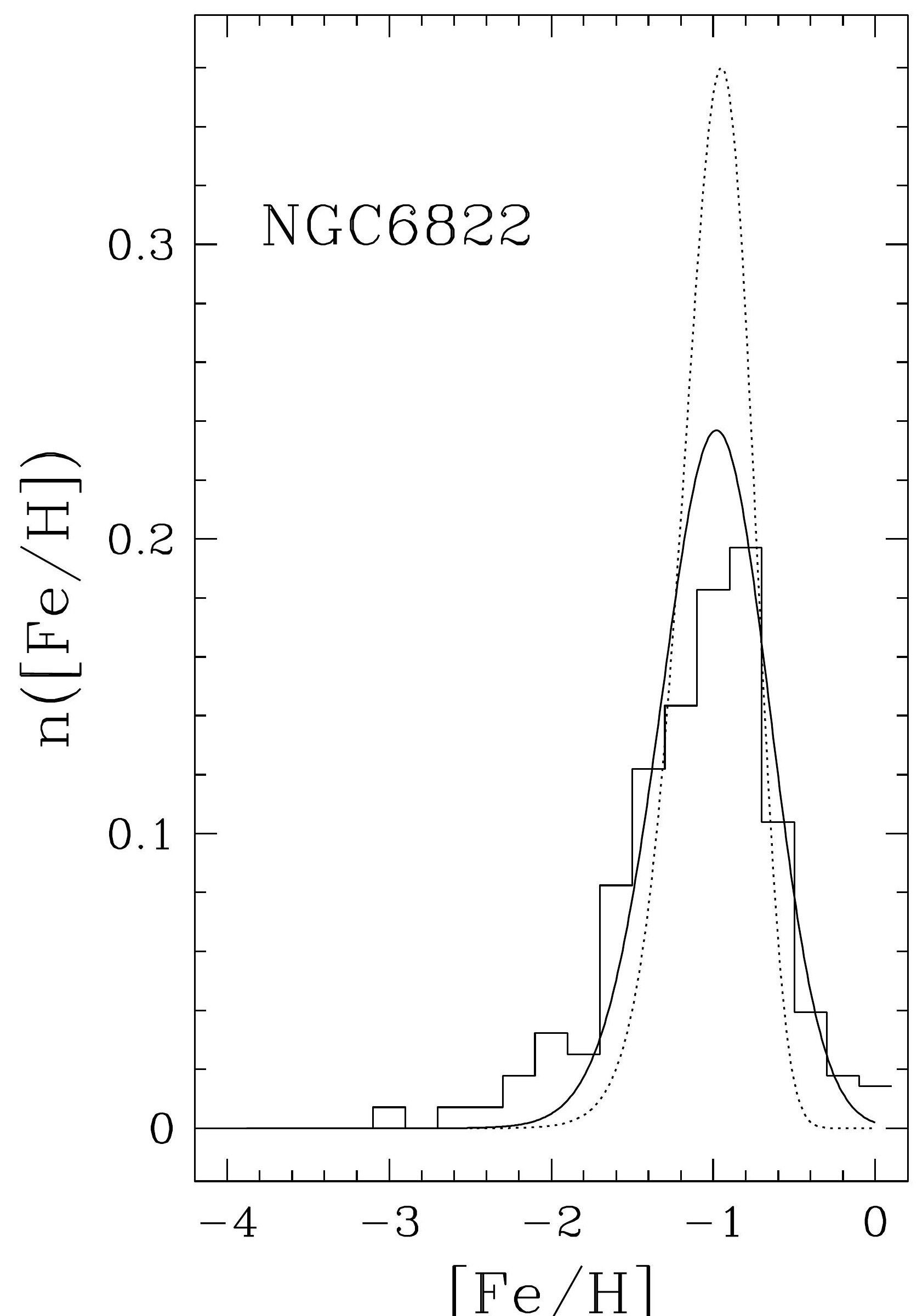




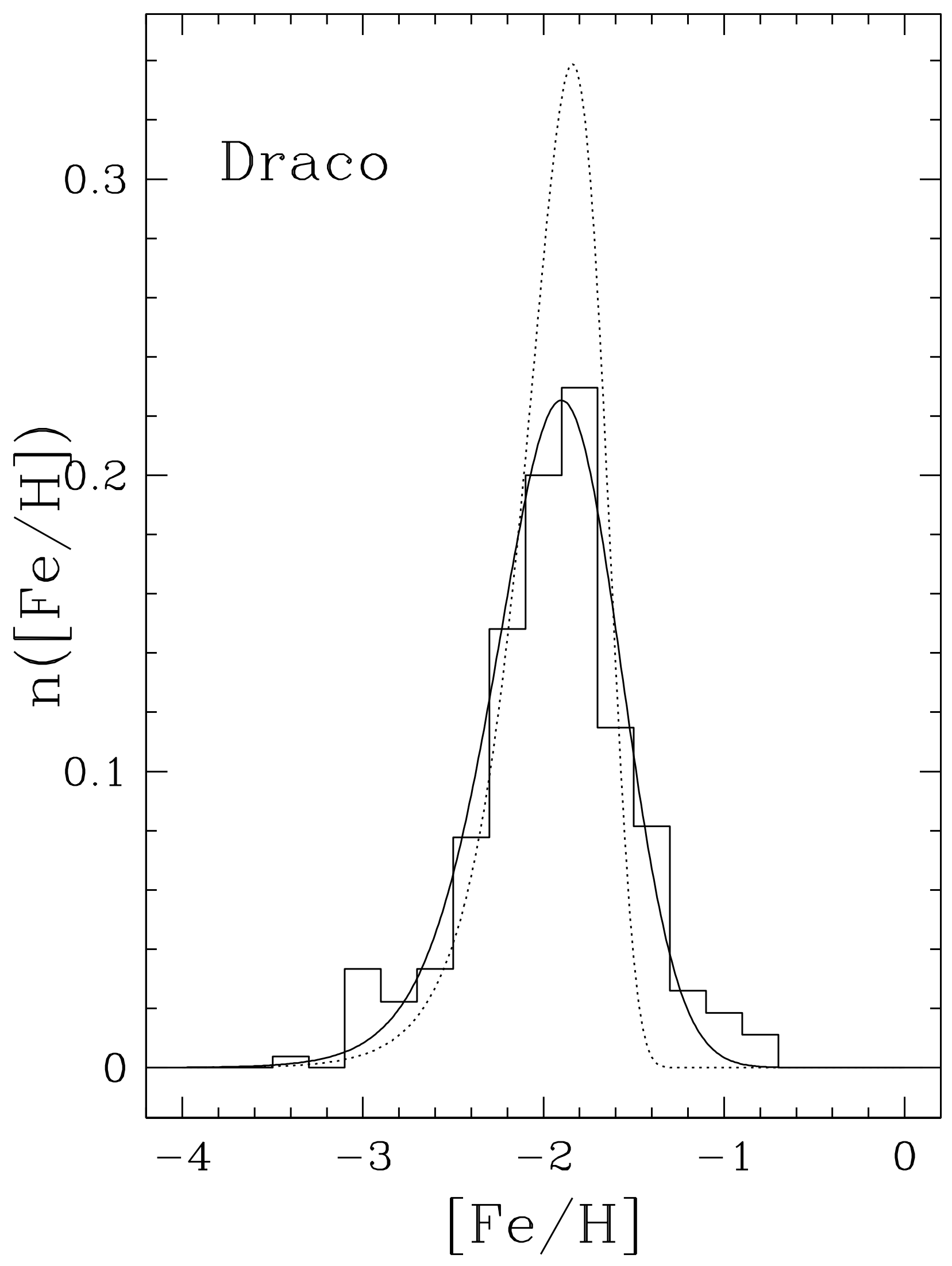

Fig. 7. $-[\mathrm{Fe} / \mathrm{H}]$ histogram for the Draco dwarf spheroidal galaxy. Dotted line - the model distribution calculated with the Gaussian kernel with $\log p_{\text {eff }}=-1.84$. Solid line - the model distribution smoothed by $\sigma=0.25$. Compare with Fig.2 